\title{
Overview on recent progress in magnetron injection gun theory and design for high power gyrotrons
}

\author{
Ioannis Pagonakis ${ }^{1, *}$, Stefano Alberti ${ }^{2}$, Konstantinos Avramidis ${ }^{1}$, Francois Legrand ${ }^{2,3}$, Gerd Gantenbein ${ }^{1}$, Jérémy \\ Genoud $^{2}$, Jean-Philippe Hogge ${ }^{2}$, Stefan Illy $^{1}$, Zisis Ioannidis ${ }^{1}$, Parth Kalaria ${ }^{1}$, Bernhard Piosczyk ${ }^{1}$, Sebastian Ruess ${ }^{1}$, \\ Tobias Ruess ${ }^{1}$, Tomasz Rzesnicki ${ }^{1}$, Minh-Quang Tran $^{2}$, Trach-Minh Tran $\dagger^{2}$, Manfred Thumm ${ }^{1}$, Ioannis Vomvoridis ${ }^{4}$, and \\ John Jelonnek ${ }^{1}$
}

${ }^{1}$ IHM, Karlsruhe Institute of Technology (KIT), D-76131 Karlsruhe, Germany

${ }^{2}$ Swiss Plasma Center (SPC), EPFL, CH-1015 Lausanne, Switzerland

${ }^{3}$ Thales Electron Devices (TED), Velizy, France

${ }^{4}$ National Technical University of Athens (NTUA), GR-15773 Athens, Greece

\begin{abstract}
The magnetron injection gun (MIG) is one of the most critical subcomponents in gyrotrons. The electron beam, which has the primary role on the gyrotron operation, is generated and configured at this part of the tube. The electron beam properties determine the excitation mode in the cavity, the power of the generated microwaves and the gyrotron efficiency. The operation of MIGs could be influenced by several factors such as trapped electrons, manufacturing tolerances, roughness of the emitter ring, emitter temperature inhomogeneity, electron beam neutralization effect, etc. The influence of many of these factors on the electron beam quality has been systematically investigated during the last years. Several novelties have been proposed in order to limit the influence of these factors on the gyrotron operation. In particular, new design criteria have been proposed for the suppression of electron trapping mechanisms, a new type of the emitter ring has been proposed to minimize the influence of the manufacturing tolerances and edge effects on the beam quality, alternative MIG design approaches have been proposed, etc. An overview of all these works will be presented here.
\end{abstract}

\section{Introduction}

The gyrotron is a millimeter and submillimeter wave source device, which is extensively used for plasma heating and current drive applications. The gyrotron operation is based on the electron cyclotron resonance maser (CRM) instability. A part of the transverse energy of the electron gyro-motion is converted to an electromagnetic wave in an open-ended cavity. The electrons emitted from a cylindrical emitter ring form an annular electron beam (HEB) under the influence of an externally applied static magnetic field. The beam quality at the entrance of the cavity plays an important role on the interaction efficiency $\eta_{e}$ which ranges between $20 \%$ and $40 \%$. For the generation of the electron beam a magnetron injection gun (MIG) is used. The geometry of the MIG subcomponents determines the quality of the HEB at the cavity. The beam electrons are produced from a heated emitter ring by temperaturelimited thermionic emission. The emitter ring is part of the cathode, which is the main subcomponent of the MIG. The electrons are accelerated by the voltage $U_{a}$ applied to the cathode and a second electrode, the anode. The convergent externally applied magnetic field guides the electrons towards the cavity, and generates the gyro-motion of the beam electrons. The MIG structure with a cathode and an anode is called a diode. More complex structures are also

*e-mail: ioannis.pagonakis@kit.edu used with the introduction of a modulation anode at an intermediate potential, controlled by an independent power supply, increasing in this way the operational parameters. The MIG structure with a modulation anode is known as a triode. Even more complex MIG structures are also used for the coaxial cavity gyrotrons [1] due to the presence of the coaxial insert which is fixed at the rear part of the MIG. Theoretical models have been developed for the estimation of the beam electron trajectories in the MIG region where a strong electric field and convergent magnetic field are applied [2]. For more accurate calculations, electron optics codes have been developed, such as EGUN [3], EPOSR [4], MICHELLE [5], etc. In Europe (EU) three electron optics codes have been developed for gyrotron MIG study and design. These are (i) the Ariadne code [6] (finite element, parallel, three and two dimensional, electrostatic, tracking and self-consistent), (ii) the ESRAY/ESPIC code [7] (finite difference, two dimensional, tracking and PIC), and (iii) the DAPHNE code [8] (finite element, two dimensional, electrostatic, tracking and self-consistent).

In reality, the operation of the gun is not as ideal as it is in the numerical simulations. The quality of the generated electron beam could be influenced by many factors which are not usually taken into account during the design phase of the MIG. Such factors are the presence of trapped electrons, which can dramatically influence the gyrotron operation, the manufacturing tolerances and misalignments, 

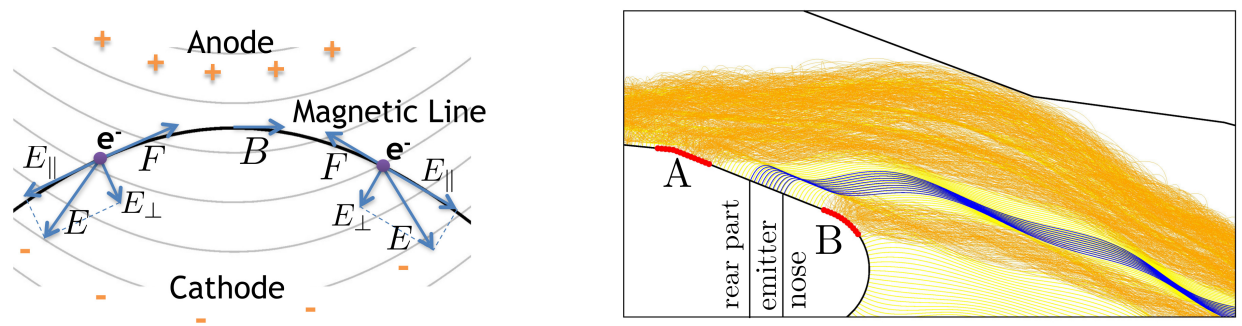

Figure 1. Equipotential lines are intersected by a magnetic field line formulating a magnetic potential well (left). Secondary electrons emitted from the region A and B are adiabatically trapped and they contribute to the generation of the harmful beam halo (right).

the emission inhomogeneity, etc. Many of these factors have been comprehensively investigated during the last years. An overview of the results of these studies will be presented here. In Sect. 2 the electron trapping mechanisms will be shortly discussed and the MIG design criteria defined for the suppression of the electron accumulation mechanisms will be presented. In Sect. 3 and Sect. 4 two important issues related to the emitter ring will be discussed. In particular, the influence of the manufacturing tolerances and the misalignment of the emitter in relation to its neighboring parts will be discussed in Sect. 3, while the effects of the azimuthal temperature non-uniformity of the emitter ring on the beam quality will be reported in Sect. 4. The paper is closing with a short summary.

\section{Electron trapping mechanisms}

During the experiments of the first industrial prototype of the EU $170 \mathrm{GHz} / 2 \mathrm{MW}$ coaxial cavity gyrotron project for ITER [9], voltage stand-off problems even with cold emitter were observed in the presence of the nominal magnetic field with a high impedance power supply. Periodic discharges were observed for voltages around $60 \mathrm{kV}$, while for voltages more than $63 \mathrm{kV}$ the leakage current exceeded the power supply current limitation. During short-pulse experiments with the electron beam, strong instabilities limited the operation of the gyrotron for accelerating voltages higher than $80-82 \mathrm{kV}$, although the nominal voltage was $90 \mathrm{kV}$.

The hypothesis of electron accumulation in the gun region was proposed for the explanation of this gyrotron behavior [10]. This hypothesis was also supported by the fact that the positions of many electron traces on the inner surface of the gyrotron components observed during the inspection of the tube after the end of the experiments, were correlated with the presence of trapped electrons. Two electron trapping mechanisms were investigated: (i) the presence of potential wells and (ii) the adiabatic trap of secondary electrons emitted from the cathode surface.

The potential well also known as Penning mechanism, can take place wherever a magnetic field line intersects the equipotential lines as shown at left of figure 1 . The electrons guided by the magnetic field line are accelerated from one side and decelerated from the other side by a force $F=-e E_{\|}$along the magnetic field line, where $E_{\|}$is the component of the electric field parallel to the magnetic

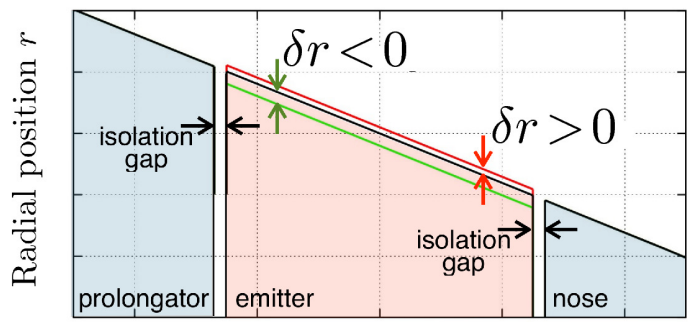

Axial position $z$

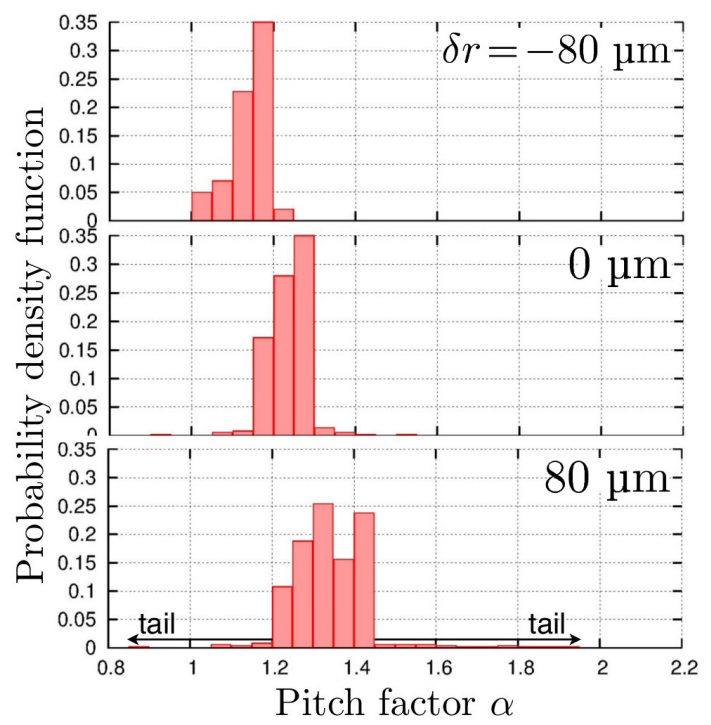

Figure 2. A small radial displacement of the emitter surface in relation to its neighboring parts (top) causes a significant variation of the pitch factor distribution (bottom).

field. Such type of potential well is usually formed at the rear part of the gun.

On the other hand, the adiabatic trap is a well known electron trapping mechanism in gyrotrons. It takes place in the region between the cathode and the cavity where the magnetic field lines converge. The transverse velocity of the beam electrons is increasing due to the conservation of the adiabatic invariant whereas the parallel velocity decreases in such a way that the total energy is conserved. As a result, some electrons can be reflected back by magnetic mirroring towards the cathode depending on their initial transverse velocity. On the other side, the decelerating 

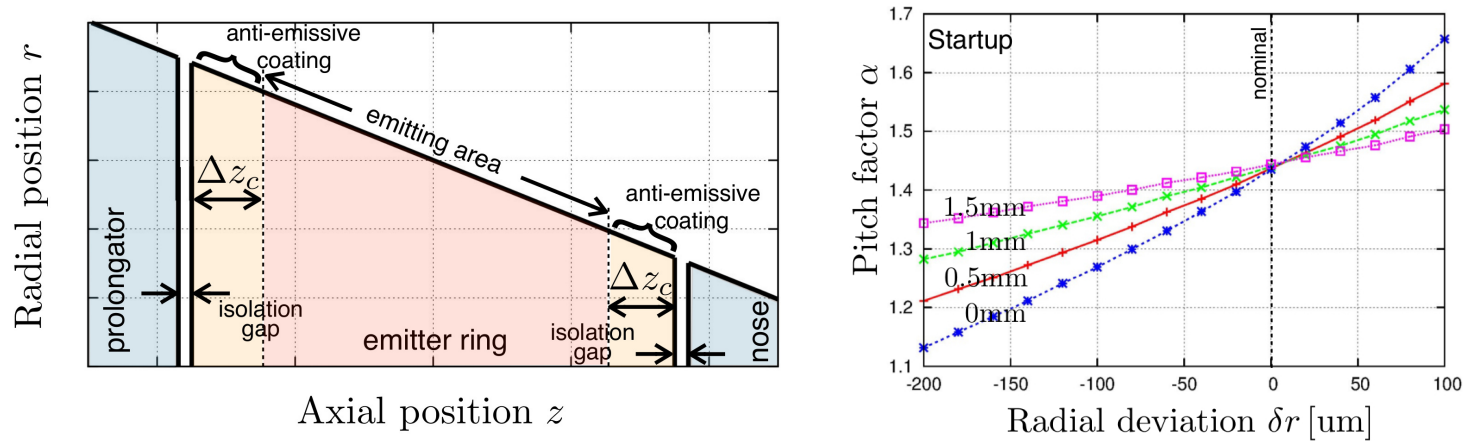

Figure 3. The new type of the emitter ring with coated edge rims (left). The variations of the average pitch factor $\alpha$ as a function of the radial deviation $\delta r$ for several values of the coating length $\Delta z_{c}$ (right).

field of the cathode reflects the electrons towards the cavity (electric mirror). It has been numerically shown that a minor number of adiabatically trapped electrons is adequate to initiate a harmful beam halo due to the adiabatic trap. These minor number of electrons bombard the cathode surface causing the emission of secondary electrons (see at right of figure 1). The properties of the secondary electrons emitted from the bombarded area of the cathode surface play a critical role in the formation of the beam halo.

Two design criteria have been determined for the suppression of the electron trapping mechanisms in MIG [11]: (i) the geometry of the MIG should be designed in such a way that the formation of potential wells is prevented, and (ii) no secondary electron emitted from the cathode surface is adiabatically trapped. These criteria have been considered for the design of the MIG of the refurbished first industrial prototype of the EU $170 \mathrm{GHz} / 2 \mathrm{MW}$ coaxial cavity gyrotron project for ITER [12] and the MIG of the EU $170 \mathrm{GHz} / 1 \mathrm{MW}$ conventional cavity gyrotron for ITER [13]. The voltage standoff of both tubes was excellent, while during the operation of the gyrotrons no instability which could be correlated with the presence of trapped electrons was observed. The proposed design criteria were also considered in other MIGs designed in EU $[14,15]$.

\section{Emitter ring tolerances}

The influence of the emitter ring alignment versus its neighboring part (cathode nose and prolongator - see figure 2-top) on the beam quality has been numerically investigated. In this study the MIG design of the EU 170 $\mathrm{GHz}, 1 \mathrm{MW}$ conventional cavity gyrotron for ITER [13] was considered.

An extreme sensitivity of the electron beam parameters in the emitter ring alignment was numerically identified. In particular, it has been shown that velocity spread and average pitch factor are significantly influenced by a minor emitter ring radial displacement. In figure 2 (bottom) the variation of the pitch factor distribution for a radial displacement $( \pm 80 \mu \mathrm{m})$ is shown. The uncertainty on the pitch factor value could influence the mode excitation and
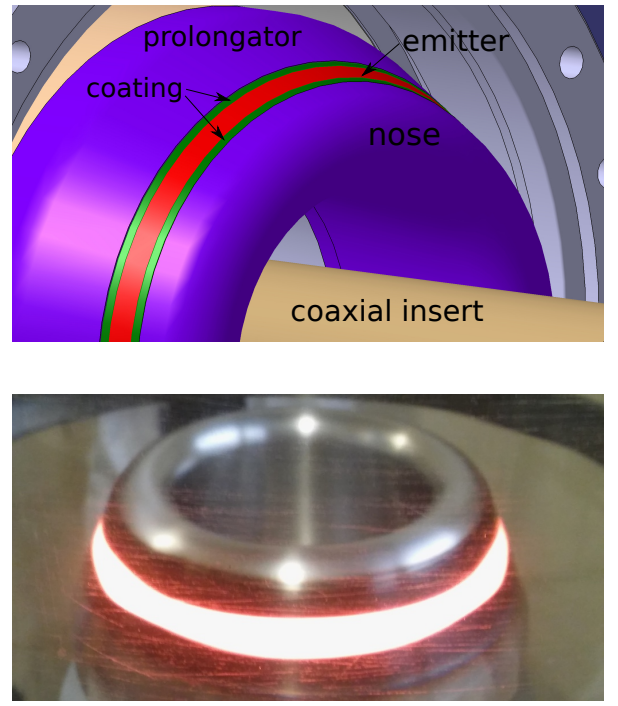

Figure 4. A three-dimensional drawing and a photo of the cathode of the new MIG with the emitter ring with the coated edge rims.

the interaction efficiency at the gyrotron cavity. Furthermore, the formation of potentially harmful long tails in the pitch factor distribution is also predicted. This high sensitivity can potentially induce a significant spread in the performance of tubes with an identical scientific design.

A possible solution of this major issue for the gyrotron MIG designs was proposed by introducing anti-emissive regions on the two edge rims of the emitter ring [16], as shown in figure 3 (left). Using this idea, it was possible to significantly suppress that sensitivity as shown in figure 3 (right).

It should be note that for the $170 \mathrm{GH} 2 \mathrm{MW} 100 \mathrm{~ms}$ coaxial cavity gyrotron [17] a new MIG has been designed and manufactured which uses for first time in EU the new type of the emitter with coated edge rims (see figure 4). Experiments with the new MIG are expected in the near future. 


\section{Emission temperature inhomogeneity}

The beam electrons are produced by thermionic emission from an emitter ring at around $900^{\circ} \mathrm{C}$. The emission in gyrotrons takes place in the temperature-limited regime. For this type of emission, the current density $J$ is defined as an exponential function of temperature (RichardsonDushman equation with Schottky correction). Therefore, a small variation of temperature may dramatically influence the current density and cause a significant inhomogeneous emission.

The purpose of this study is to evaluate whether a typical specified tolerance of $\pm 15^{\circ} \mathrm{C}$ for the temperature azimuthal inhomogeneity of the emitter ring has a negative impact on the gyrotron operation. For the definition of the current density inhomogeneity a similar procedure as in ref. [18] was followed. In particular, the current density was defined as $J(\varphi)=J_{0}(1+\epsilon \cos (n \varphi))$, where $\epsilon$ is the amplitude and $n$ is the harmonic of the inhomogeneity. Three dimensional simulations were performed for the EU $170 \mathrm{GHz}, 1 \mathrm{MW}$ conventional cavity gyrotron for ITER using different values of the harmonic number $n$. The inhomogeneity amplitude $\epsilon$ was set equal to 0.5 . This value approximates an azimuthal current density inhomogeneity which corresponds to the temperature non-uniformity of the order of $\pm 15^{\circ} \mathrm{C}$.

The beam parameter which is mostly influenced by the current density inhomogeneity is the kinetic energy spread. As shown in figure 5, the kinetic energy of the electrons (left) and the kinetic energy distributions (right) are significantly different from the homogeneous emission case shown in black. The energy spread which in the ideal case is only $0.1 \%$, at the worst case scenraio of $n=1$ becomes $2 \%$. However, preliminary mono-mode[19] and multi-mode[20] cavity simulations predict that the influence of such a higher energy spread does not have an important impact neither on the excitation and the stability of the operating mode nor on the interaction efficiency. More details on this work will be presented to the scientific community in the near future.

\section{Summary}

Three important issues related to MIG theory and design were presented: (i) Two electron trapping mechanisms were discussed while design criteria were proposed for the suppression of the electron trapping mechanisms. (ii) The influence of a possible emitter ring radial displacement in relation to its neighboring parts on the beam quality was numerically investigated. The results show that such a radial displacement significantly influences the gyrotron operation. A new type of emitter ring with coated edge rims could suppress that sensitivity. (iii) The influence of the specified emitter ring temperature inhomogeneity on the beam quality was also shortly discussed.

\section{Acknowledgments}

Part of this work was supported by Fusion for Energy under Contract Nos. F4E-GRT-008, F4E-GRT-049, F4EGRT-432, and F4E-GRT-553 to the European Gyrotron
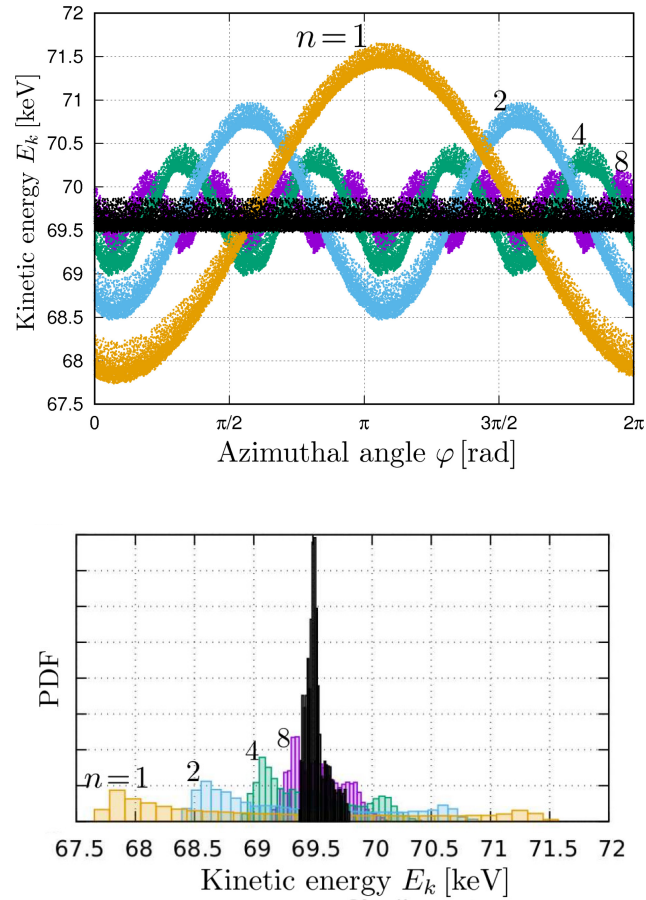

Figure 5. Kinetic energy of the beam electrons at top and the kinetic energy distribution for several values of inhomogeneity harmonic $n$ at bottom.

Consortium (EGYC). EGYC is collaboration among SPC, Switzerland; KIT, Germany; HELLAS, Greece; and IFPCNR, Italy. In addition, a part of this work has been carried out within the framework of the EUROfusion Consortium and has received funding from the Euratom research and training program 2014-2018 under grant agreement No 633053. The views expressed in this publication do not necessarily reflect the views of F4E or the European Commission.

\section{References}

[1] B. Piosczyk, et al., IEEE Trans. Plasma Science 32, 853 (2004).

[2] S.E. Tsimring, Electron beams and microwave vacuum and electronics (Wiley, New Jersey, 2007)

[3] W.B. Herrmannsfeldt, EGUN - An Electron Optics and Gun Design Program (Stanford Linear Accelerator Center, 1988)

[4] V.K.Lygin, V.N.Manuilov, Sh.E.Tsimring, Beams 96, 385-388 (1996)

[5] J.J. Petillo, E.M. Nelson, et al. IEEE Trans. on Electron Devices 52, 742-748 (2005)

[6] I.Gr. Pagonakis, J.L. Vomvoridis, IRMMW-THz, $657-$ 658 (2004)

[7] S. Illy, J. Zhang, J. Jelonnek, Gyrotron electron gun and collector simulation with the ESRAY beam optics code, IEEE IVEC (2015)

[8] T.M. Tran, et all, EPS-APS (1994)

[9] J.P. Hogge, S. Alberti, et al., Fusion Science and Technology 55, 204-210 (2009) 
[10] I. Gr. Pagonakis,et al., Gun Design Criteria for the Refurbishment of the First Prototype of the EU 170GHz/2MW/CW Coaxial Cavity Gyrotron for ITER, IRMMW-THz (2009)

[11] I. Gr. Pagonakis, B. Piosczyk, et al., Physics of Plasmas 23, 23105 (2016)

[12] S. Kern, et al., EPJ Web of Conference 32, 04009 (2012)

[13] J. Jelonnek, F. Albajar, et al., IEEE Trans. on Plasma Science 42, 1135 (2014)

[14] S. Ruess, I.Gr. Pagonakis, et al., IEEE Trans. on Electron Devices 63, 2104-2109 (2016)
[15] J. Franck, I. et al., GeMiC, 260-263 (2015)

[16] I.Gr. Pagonakis, S. Illy, M. Thumm, Physics of Plasmas 23, 83103 (2016)

[17] S. Ruess, et al., 47th EuMC, 2017, 860-863 (2017)

[18] J. Gr. Pagonakis, J. L. Vomvoridis, IEEE Trans. on Plasma Science 32, 890 (2004)

[19] F. Braunmueller, T.M. Tran, et al., Physics of Plasmas 22, 063115 (2015).

[20] K. A. Avramides, et al., EPJ Web of Conference 32, 04016 (2012) 